\title{
Comparaison entre les prélèvements pastoraux déterminés par des lectures de végétation et l'estimation des quantités ingérées par des brebis sur trèfle souterrain et végétation steppique
}

\author{
G Molénat 1, T Adama 2, P Lapeyronie 1, J Gouy 1 \\ IINRA-ENSA.M, Unité de Zootechnie Méditerranéenne, 34060 Montpellier, France ; \\ 2School of Agriculture, Fed Univ of Technology, PMB 65 Ninna, Nigéria
}

L'estimation des quantités ingérées par les ovins au pâturage est un élément clé de l'analyse des systèmes herbagers ou pastoraux. Le travail présenté ici a consisté à développer une méthode d'évaluation des quantités de fourrages pâturées par les ovins, basée sur des mesures de végétation avant et après pâturage. Par rapport aux méthodes "classiques" faisant appel à la récolte totale des fèces ou à des observations directes sur l'animal nous en espérons une meilleure adaptation à des situations complexes : troupeaux gardiennés, pâturage sur plusieurs ressources fourragères.

La méthode proposée est basée sur une lecture de transects en termes d'espèces végétales (Daget et Poissonet, 1971, An Agr, 22, 5-41) et de hauteur des végétaux recensés (Heady, 1957, Ecology, 38, 313-320). Sur des durées de pâturage courtes (4 jours) permettant de minimiser les biais dus à la croissance végétale, la végétation disparue représente le prélèvement effectué par les brebis.

Ce travail a été conduit dans la plaine méditerranéenne de la Crau sur deux types de couverts végétaux, un trèfle souterrain en culture sèche et une végétation pastorale spontanée steppique $(70$ à $75 \%$ de graminées, 15 à $30 \%$ de composées et espèces diverses, 1 à $2 \%$ de légumineuses). Trois mesures ont été réalisées au printemps
1993 simultanément à des estimations de quantités ingérées basées sur la récolte totale des fèces émises par 5 brebis du troupeau et sur la digestibilité du fourrage obtenue soit par l'index de l'azote fécal (Lancaster, 1954, NZJ Sci Techn, 36, 15-18) soit par des digestibilités $48 \mathrm{~h}$ in situ de rations reconstituées simulées (Lapeyronie, Adama, Hubert, Gouy, 1995, IV Symp Int Nutr Herb).

La quantité de matière organique $(\mathrm{MO})$ ingérée estimée a été en moyenne de $978 \mathrm{~g} / \mathrm{j} / \mathrm{brebis}$ sur le trèfle souterrain avec une digestibilité déduite des digestibilités $48 \mathrm{~h}$ in situ; elle était supérieure de $20 \%$ avec l'index fécal azote $(1177 \mathrm{~g})$. Le prélèvement estimé le long des transects, $895 \mathrm{~g}$ de $\mathrm{MO}$ en moyenne, était légèrement inférieur à l'ingestion observée à partir des digestibilités $48 \mathrm{~h}$ in situ. Sur la végétation steppique, la hiérarchie des quantités de $M O$ ingérées est la même : $1039 \mathrm{~g} / \mathrm{j}$ avec les digestibilités $48 \mathrm{~h}$ in situ et $1358 \mathrm{~g} / \mathrm{j}$ avec l'index fécal azote (écart de $30 \%)$; le prélèvement le long des transects fournit des valeurs plus élevées : $1493 \mathrm{~g} / \mathrm{j}$ en moyenne.

Les prélèvements estimés par les lectures de végétation le long des transects sont cohérents avec les estimations de quantités ingérées et peuvent s'y substituer. Dans le cas de la végétation steppique, il nous semble que l'estimateur retenu à partir des digestibilités $48 \mathrm{~h}$ in situ sous-estime la digestibilité.

\begin{tabular}{|c|c|c|c|c|c|c|}
\hline & \multicolumn{3}{|c|}{ trèfle souterrain } & \multicolumn{3}{|c|}{ végétation steppique } \\
\hline & mi mars & mi avril & mi mai & mi mars & mi avril & mi mai \\
\hline \multicolumn{7}{|l|}{ MO ingérée (g/j) } \\
\hline à partir de in situ & 846 & 1083 & 1003 & 962 & 1054 & 1102 \\
\hline à partir de $\mathrm{N}$ fécal & 1049 & 1355 & 1127 & 1416 & 1386 & 1273 \\
\hline \multicolumn{7}{|l|}{ MO prélevée (g/j) } \\
\hline à partir des transects & 724 & 1035 & 924 & 1529 & 1557 & 1402 \\
\hline
\end{tabular}

